\title{
Genderforschung - gendergerechte Karriereplanung und Spitalstrukturen
}

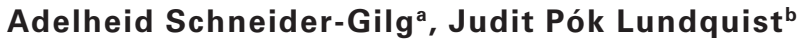

${ }^{a}$ Dr. med., Innere Medizin, FMH, Präsidentin medical women switzerland; ' ${ }^{b}$ Dr. med., Gynäkologie/Geburtshilfe, FMH, Vorstandsmitglied medical women switzerland

Die Jahrestagung der mws - medical women switzerland (Vereinigung der Schweizer Ärztinnen) stand unter dem immer noch hochaktuellen Thema "Gendergerechte Karriereplanung - Genderforschung und ihre Konsequenzen auf Karriereplanung und Spitalstrukturen». Die Referentinnen haben neue Erkenntnisse aus der Genderforschung präsentiert, Top-Karrieren aufgezeigt und Parallelen zur Wirtschaft gezogen.

Tatsache ist, dass nach wie vor eine relevante genderbedingte Diskrepanz in der Karriereentwicklung besteht, wenn es um die Besetzung von höheren Kaderstellen geht: Nur 13\% der Stellen für leitende Positionen, insbesondere für Chefärztinnen und in akademischen Forschungsbereichen, sind von Frauen/ Ärztinnen besetzt. Oder anders gesagt: Bei Beförderungen werden Frauen unterproportional in höhere Positionen gewählt, signifikant weniger häufig als es dem bestehenden Frauenanteil im Beruf entsprechen würde. Die wichtigsten Gründe dafür konnten wie folgt verortet werden.

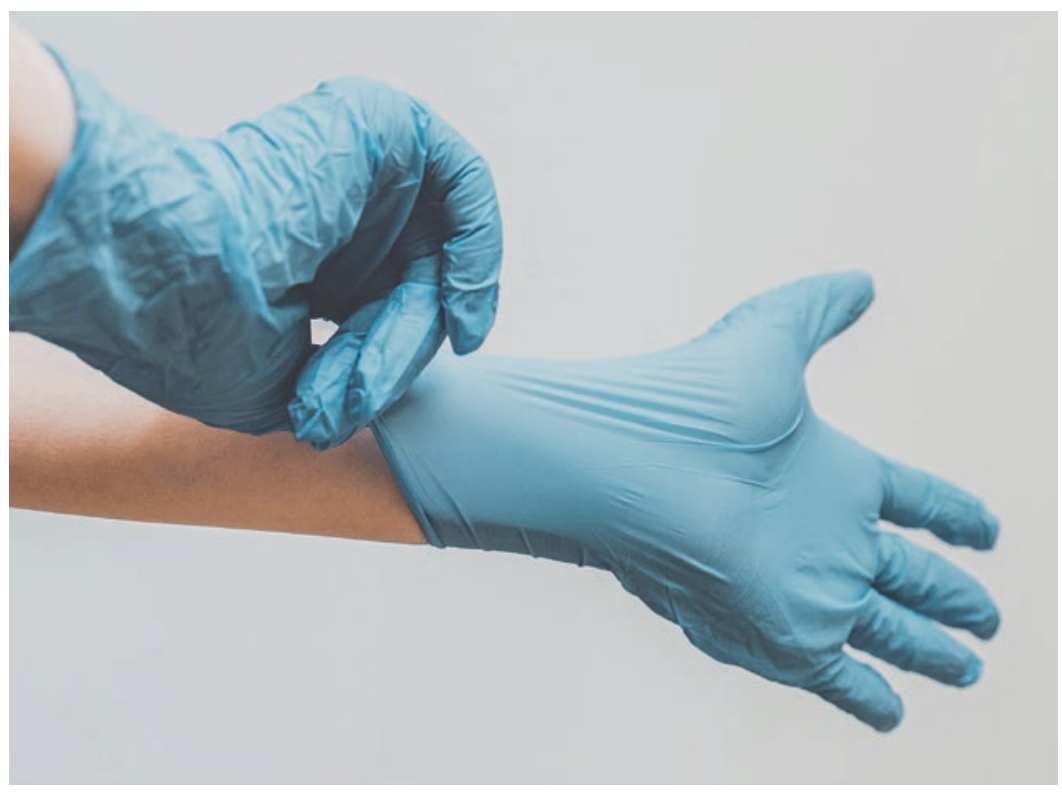

Ärztinnen: Im Beruf engagiert, aber zu selten befördert.

\section{Genderbedingte Diskrepanz in der Karriereentwicklung - die Gründe}

- Die Norm der Vollzeitstelle: Kaderpositionen sind in der Regel Vollzeitstellen. Die Beförderungen in Kaderpositionen finden in der Regel zwischen dem 30. und 40. Lebensjahr statt, einer Zeit, in welcher viele Frauen insbesondere aus familiären Gründen zu Teilzeitarbeit übergehen beziehungsweise mutterschaftsbedingte Abwesenheiten aufweisen. Entsprechend erfahren sie - im Vergleich zu ihren männlichen Kollegen, die häufiger ihre hundertprozentige Arbeitskraft anbieten können - im Auswahlverfahren für Kadermitarbeitende bereits im Anfangsstadium des Aufstiegs auf der Karriereleiter einen bedeutenden Nachteil.

- Unterschiedliche Vorstellungen von Führung: Während in manchen Betrieben eine direkte Führung mit klaren hierarchischen Strukturen herrscht, ist in anderen Betrieben die indirekte Führung die Norm, mit Betonung der Teamarbeit, von Wertschätzung und gegenseitigem Vertrauen. Indirekte Führung ist für Frauen, wie Untersuchungen gezeigt haben, attraktiver als das direkte Führungsmodell, das jedoch immer noch weitverbreitet ist. Entsprechend ist in Betrieben mit direkter, hierarchischer Führung schon die Anzahl der sich um Kaderstellen bewerbenden Frauen tiefer als diejenige der Männer.

- Beförderung aufgrund der Expertise: Beförderungen geschehen in der Regel aufgrund des Wissens und des Könnens, also der fachlichen Expertise. Führungskompetenzen spielen oft eine untergeord- 
mws - medical women switzerland - ärztinnen schweiz

Gegründet 1922 unter dem Namen «Vereinigung Schweizer Ärztinnen» VSÄ. Heute kann die mws auf knapp 1000 Kolleginnen zählen.

Kompetent und mit gezielt weiblichem Blick vertritt die mws - medical women switzerland (ärztinnen schweiz) als einziger Verband der Schweiz die Interessen von Medizinstudentinnen, Ärztinnen in Weiterbildung und Ärztinnen aller Fachrichtungen und Positionen in Spitälern, Instituten und Praxen aus jeder Region - mit oder ohne aktuelle Berufsausübung.

\section{Unser Credo}

- Wir machen uns stark für individuell zugeschnittene Berufs- und Karrierewege und die Vereinbarkeit von beruflichem, sozialem und familiärem Engagement. Unser Netzwerk berät Medizinstudentinnen und junge Ärztinnen bei Laufbahnfragen, verbindet und unterstützt Ärztinnen in Praxen sowie Führungspositionen und schafft Synergien.

- Wir setzen uns dafür ein, dass die weibliche Perspektive in Ausbildung, Beruf, Standespolitik und Forschung anerkannt wird, und Ärztinnen in allen Fachrichtungen und Führungspositionen adäquat vertreten sind. Jede Ärztin soll dazu ermutigt werden, ihr weibliches Selbstverständnis im Beruf einzubringen.

- Wir analysieren gesellschaftliche und gesundheitspolitische Entwicklungen hinsichtlich ihrer Bedeutung für Ärztinnen ebenso wie für die weibliche Bevölkerung

- Überall dort, wo Frauenanliegen zu wenig berücksichtigt werden, sind wir besonders aktiv. Nur wenn unsere Überzeugungsarbeit in allen relevanten medizinischen Institutionen und im öffentlichen Bewusstsein greift, können wir unsere Anliegen zum Wohl aller Beteiligten langfristig durchsetzen.

Für mehr Informationen besuchen Sie unsere Homepage: www.medicalwomen.ch

nete Rolle, was problematisch ist. Viele Frauen sind in ihrem Beruf zwar bestens qualifiziert und erfüllen sämtliche fachlichen Voraussetzungen, um eine Kaderposition zu übernehmen. Ein Nachteil liegt bei vielen Frauen aber darin, dass sie aufgrund von Teilzeit nicht für Beförderungen in Betracht gezogen werden oder, und das wiegt schwerer, dass sie sich, im Gegensatz zu männlichen Kollegen, die Führungsaufgabe nicht zutrauen.

\section{Minimierung und Eliminierung der gender- bedingten Diskrepanz - Lösungsansätze}

Um eine Karriere, einen beruflichen Aufstieg im Bereich Spitäler/Kliniken und akademischer Forschung anstreben zu können und zu wollen, braucht es für Frauen wie für Männer sowohl ein geeignetes Umfeld als auch, nebst Fachwissen, persönliche Eigenschaften wie Beharrlichkeit, Eigenverantwortung, Mut zur Entscheidung, Selbstreflexion und den Willen, Verantwortung für andere Menschen und einen Betrieb zu übernehmen.

Für ein geeignetes Umfeld sind ein gutes Arbeitsklima, Fairness und Transparenz und ein inklusives Arbeits- umfeld wünschenswert. Die obgenannten, persönlichen Eigenschaften sind nicht allen Menschen, weder Männern noch Frauen, auf Anhieb gegeben. Sie können aber gefördert und erlernt werden. Beispielsweise besteht die Möglichkeit, sich im Rahmen einer Weiterbildung Führungsqualitäten und auch die nötigen Softskills, die von Kadermitarbeitenden erwartet werden, anzueignen. Zudem darf Teilzeitarbeit nicht per se als Karrierekiller wirken. Falls Karriere tatsächlich eine konsumierende Vollzeitstelle verlangen sollte, so können und sollen alternative Arbeitsmodelle versucht werden, wie zum Beispiel ein Jobsharing.

\section{Weiterbildung für Assistenzärztinnen}

Es ist dringend nötig, dass Frauen (und auch Männer) in ihrer Assistenzzeit die Möglichkeit einer Weiterbildung hinsichtlich Karriereplanung erhalten, wenn sie dies wünschen. Die mws unterstützt deshalb aktuell ein auf Initiative von Frau Prof. Dr. Gudrun Sander von der Universität St.Gallen entstehendes Projekt für ein zweijähriges Begleitprogramm für Assistenzärztinnen: Der Fokus dieses Programms wird darauf liegen, den Assistenzärztinnen Hilfestellung beim Treffen von individuellen Karriereentscheidungen zu geben und sie mit benötigten Informationen und Entscheidungshilfen zu unterstützen. Das Begleitprogramm wird mehrheitlich online durchgeführt und kann deshalb zeitlich unabhängig und in-

\section{«Teilzeitarbeit darf nicht per se als Karriere-} killer wirken.»

dividuell absolviert werden. Starten soll es in der zweiten Jahreshälfte 2021. Das Programm wird massgeblich von den Deutschschweizer Universitätsspitälern Basel, Bern und Zürich mitfinanziert. Wir freuen uns, dass die mws dieses Projekt mit Beratung und später im Mentoringprogramm tatkräftig unterstützen kann.

Bildnachweis

Unsplash/Clay Banks

\section{Das Wichtigste in Kürze}

- Nur 13\% der Stellen für leitende Positionen sind von Frauen/ Ärztinnen besetzt.

- Aufgrund von Teilzeitarbeit werden viele Frauen nicht für Führungspositionen in Betracht gezogen.

- $\quad$ Ein neues zweijähriges Begleitprogramm kann Assistenz ärztinnen bei der Karriereplanung unterstützen. 